\title{
NON-LINEAR VARIATION OF THE BETA FUNCTION WITH MOMENTUM
}

\author{
George Parzen
}

\section{DISCLAIMER}

\begin{abstract}
This report was prepared as an account of work sponsored by an agency of the United States Government. Neither the United States Government nor any agency thereof, nor any of their employecs, makes any warranty, express or implied, or assumes any legal liability or responsibility for the accuracy, completeness, or usefulness of any information, apparatus, product, or process disclosed, or represents that its use would not infringe privately owned rights. Reference herein to any specific commercial product, process, or service by trade name, trademark, manufacturer, or otherwise does not necessarily constitute or imply its endorsement, recommendation, or favoring by the United States Government or any agency thereof. The views and opinions of authors expressed herein do not necessarily state or reflect those of the United States Government or any agency thereof.
\end{abstract}

\section{July 1983}

\section{ACCELERATOR DEPARTMENT}

\section{BROOKHAVEN NATIONAL LABORATORY UPTON, LONG ISLAND, NEW YORK 11973}




\section{DISCLAIMER}

Tlis report was prepared as an accounl of work sponsored by an agency of the ('inited States Government. Neither the I 'nited States Government nor any agency thereof, nor any of their employees, nor any of their contractors, subcontractors. or theil employees, makes any warranty, express or implicd, or assumes any legal liability or responsibility ferr the accuracy, completemess, m usefulness of any infofmation, apparalus. product, ot process disclosed. or tepresents that is use would not infringe privately ow'ned rights. Reference herein io any specific commercial product, process, or service by trade name, trademark, matnufacturer, or otherwise, does not necessarily constitute or implv its endorsement, recommendation, or favoring by the ('nited States Covernment or any agency. contractor or sabcontracter thereol. The view's and opinions of authors expressed herein do not necessarily state or reflect those of the l'nited States Covernment or any agency. ceitutractor or subcontractor therec:

Printed in the linited States of America

Available from

National Technical Information Service

U.S. Department of Commerce

$\mathbf{5 2 8 5}$ Port Royal Road

Springfield, VA 22161

NTIS price codes:

Printed Copy: A02; Microfiche Copy: A01 


\section{Abstract}

A theory is presented for computing the non-linear dependence of the $B-$ functions on momentum. Resulte are found for the quadratic term. The results of the theory are compared with computed results. A procedure is proposed for computing the strengths of the sextupole correctors to correct the dependence of the $B$-function on momentum. 


\section{Introduction}

The dependence of the $R$-function on particle momentum, $\Delta p / p$, may depart appreciably from being linear in $\Delta p / p$ for some lattices. This paper finds results for the quadratic term in the 8 -function dependence on $\Delta p / p$.

For the case where the operating $\nu$-value is close to a half-integer value, and the momentum depandence of the $\beta$-function is dominated by the corresponding half-integer stop band, it has been found that the maximum variation in the $B$ function with $\Delta p / p$ can be written as

$$
\frac{\Delta \beta}{\beta}=\left(\frac{\Delta \beta}{\beta}\right)^{(1)}+\left(\frac{\Delta \beta}{B}\right)^{(2)}
$$

$(\Delta \beta / B)^{(1)}$ is the term which is linear in $\Delta p / p$,

$$
\left(\frac{\Delta B}{\beta}\right)^{(1)}=\frac{2}{n-2 v}\left|\Delta v_{2}\right|,
$$

where $n$ is the integer closest to $2 v$, and $\Delta v_{2}$ is the half-width of the halfinteger stop band at $v=n / 2 .(\Delta B / \beta)^{(2)}$ is the term which is quadratic in $\Delta \mathrm{p} / \mathrm{p}$,

$$
\left(\frac{\Delta B}{\beta}\right)^{(2)}=\frac{3}{(n-2 v)^{2}} c \frac{\Delta p}{p}\left|\Delta \nu_{2}\right|+\frac{1}{(n-2 v)^{2}}\left|\Delta \nu_{2}\right|^{2},
$$

where $\mathrm{C}$ is the chromaticity, $\mathrm{C}=\mathrm{p} d v / \mathrm{dp}$. The above results are derived in Section 2.

The above results of the theory were compared with computed results using the SYNCH program for a lattice with a rapid dependence of the $B$-function on momentum. Using the above theoretical results, a least squares procedure is 
proposed for choosing the sextupole strengths that will minimize the variation of the $\beta$-function with momentum.

\section{Theory of Non-Linear $B \times(p), B(p)$}

Courant and Snyder ${ }^{1}$ presented the linear theory for the change in the Bfunction due to a change in the gradient function. They showed that the change in $B$-function $\Delta B$ caused by a change in the gradient function $\Delta R$, obeys the equation

$$
\begin{gathered}
\frac{d^{2}}{d A^{2}}\left(\frac{\Delta B}{B}\right)+4 \nu^{2}\left(\frac{\Delta B}{B}\right)=-v^{2} B^{2} \Delta K, \\
\Delta K=\Delta G / B \rho, \\
d A=d s / U B .
\end{gathered}
$$

Equation (2.1) can be used to compute the linear variation of $\beta$ with momentum by putting

$$
\Delta K=\left(-G \Delta p / p+2 B_{2} x_{p} \Delta p / p\right) / B O
$$

where $B_{2}(s) x^{2}$ is the sextupole field and $x_{p}(s)$ is the horizontal closed orbit dispersion function.

To obtain an equation for $\Delta B$ which includes higher order terms in $\Delta k$, we start from the equation for $b=\beta^{1 / 2}$,

$$
\frac{d^{2}}{d s^{2}} b+k b-\frac{1}{b^{3}}=0 .
$$

Letting $\mathrm{K} \rightarrow \mathrm{K}+\Delta \mathrm{K}$, and $\mathrm{b} \rightarrow \mathrm{b}+\Delta \mathrm{b}$, and keeping up to second order terms in $\Delta \mathrm{b}$ and $\Delta k$, one finds the equation for $\Delta b$ 


$$
\frac{d^{2}}{d s^{2}} \Delta b+\left(k+\frac{3}{b^{4}}\right) \Delta b=-b \Delta k-\Delta k \Delta b+\frac{6}{b^{3}}\left(\frac{\Delta b}{b}\right)^{2}
$$

Now we carry out a transformation similar to the usual orbit theory transformation $n=x / \beta^{1 / 2}$. We put

$$
\zeta=\Delta \mathrm{b} / \mathrm{B}^{1 / 2}=\Delta \mathrm{b} / \mathrm{b}
$$

Let $W$, the betatron phase function, obey $d \psi / d s=1 / B=1 / b^{2}$, then

$$
\begin{aligned}
\frac{d}{d s} \Delta b & =\frac{d b}{d s} \zeta+b \frac{d \zeta}{d s} \\
& =\frac{d b}{d s} \zeta+\frac{1}{b} \frac{d \zeta}{d \downarrow} \\
\frac{d^{2}}{d s^{2}} \Delta b & =\frac{d^{2} b}{d s^{2}} \zeta+\frac{d b}{d s} \frac{d \zeta}{d \downarrow} \frac{1}{b^{2}}+\frac{d^{2} \zeta}{d: t^{2}} \frac{1}{b^{3}}-\frac{d \zeta}{d \downarrow} \frac{1}{b^{2}} \frac{d b}{d s} \\
& =\left(-K b+\frac{1}{b^{3}}\right) \zeta+\frac{d^{2} \zeta}{d \psi^{2}} \frac{1}{b^{3}} \cdot
\end{aligned}
$$

Putting this result into Eq. (2.4) for $\Delta b$, one finds thi equation for $\zeta=\Delta b / b$

$$
\frac{1}{b^{3}} \frac{d^{2} \zeta}{d b^{2}}+\left(-K b+\frac{1}{b^{3}}\right) \zeta+\left(K+\frac{3}{b^{4}}\right) b \zeta=-b \Delta K-b \Delta K \zeta+\frac{6}{b^{3}} \zeta^{2},
$$

or

$$
\frac{d^{2} \zeta}{d A^{2}}+4 \nu^{2} \zeta=-\nu^{2} b^{4} \Delta K-\nu^{2} \Delta K b^{4} \zeta+6 \nu^{2} r^{2}
$$

Eq. (2.7) is correct to second order in $\Delta \mathrm{K}$. It may be interesting to note that one can also obtain the exact equation by replacing $6 \zeta^{2}$ by $1 /(1+\zeta)^{3}$ $-1+3 \zeta$, which gives 


$$
\frac{d^{2} \zeta}{d A^{2}}+4 v^{2} \zeta=-v^{2} b^{4} \Delta K-v^{2} \Delta R b^{4} \zeta+v^{2}\left\{\frac{1}{(1+\zeta)^{3}}-1+3 \zeta\right\}
$$

Eq. (2.7) reduces to the linear Courant-Snyder equation, Eq. (2.1), if one drops the second order terms on the right hand side.

Eq. (2.7) can be solved by successive approximations to find $\Delta B / \beta$ up to second order terms. The linear solution $\zeta^{(1)}$ can be found by neglecting the second order terms. This gives the solution found by Courant and Snyder.

$$
\begin{gathered}
\zeta^{(1)}=-\frac{1}{4 \sin (2 \pi \nu)} \int \mathrm{ds} s_{0} \cos \left(2 \pi \nu-\left|\psi(s)-\psi\left(s_{0}\right)\right|\right) B\left(s_{0}\right) \Delta K\left(s_{0}\right), \\
\Delta R=\left(-G \Delta p / p+2 B_{2} x_{p} \Delta p / p\right) / B p .
\end{gathered}
$$

Note that $\zeta^{(1)}=\Delta B / 2 B$ to first order.

$$
\begin{aligned}
& \text { An alternative solution for } \zeta^{(1)} \text { is } \\
& \zeta^{(1)}=\sum_{\mathrm{m}} \zeta_{\mathrm{m}}^{(1)} \mathrm{e}^{\mathrm{imA}}, \\
& \zeta_{m}^{(1)}=\frac{\left(v^{2} b^{4} \Delta R\right)_{m}}{m^{2}-4 v^{2}}, \\
& \left(v^{2} b^{4} \Delta K\right)_{m}=2 v \frac{1}{4 \pi} \int d s B \Delta R e^{-i m A}, \\
& \Delta R=\left(-G \Delta p / P+2 B_{2} X_{p} \Delta p / p\right) / B P .
\end{aligned}
$$

If $2 \mathrm{~V}$ is close to the integer $\mathrm{n}$, then the doninant term is 


$$
\begin{gathered}
\zeta_{n}^{(1)}=\frac{\left(v^{2} b^{4} \Delta K\right) n}{n^{2}-4 v^{2}}, \\
\zeta_{n}^{(1)}=\frac{2 v}{n^{2}-4 v^{2}} \Delta v_{2}, \\
\Delta \nu_{2}=\frac{1}{4 \pi} \int d s B \Delta \operatorname{Ke}^{-i n^{A} .}
\end{gathered}
$$

$\Delta \nu_{2}$ is the halt-width of the stopband at $v=n / 2$, driven by the off momentum gradient $\Lambda \mathrm{K}=\left(-\mathrm{G} \Delta \mathrm{p} / \mathrm{p}+2 \mathrm{~B}_{2} \mathrm{X}_{\mathrm{p}} \Delta_{\mathrm{p}} / \mathrm{p}\right) / \mathrm{BO}$.

The second order term in $\zeta$ way be found by putting $\zeta=\zeta^{(1)}+\zeta^{(2)}$, and solving the following equation for $\zeta^{(2)}$.

$$
\frac{d^{2}}{d A^{2}} \zeta^{(2)}+\left(4 v^{2}+v^{2} \Delta k b^{4}\right) \zeta^{(2)}=6 v^{2} \zeta^{(1)^{2}}
$$

The $\nu^{2} b^{4} \Delta K$ term will shift the $\nu$ value by $\Delta v$ given by

$$
\begin{aligned}
8 v \Delta v & =\left(v^{2} b^{4} \Delta K\right)_{0} \\
& =2 v \subset \wedge P / P,
\end{aligned}
$$

or

$$
\Delta v=\frac{1}{4} \mathrm{C} \Delta \mathrm{P} / \mathrm{P} \text {, }
$$

$$
C=\frac{1}{4 \pi} \Gamma_{\mathrm{ds} B} B \mathrm{~K},
$$

where $C$ is the chromaticity $C=p d v / d p$.

We rill write Eq. (2.12) as

$$
\frac{d^{2}}{d A^{2}} \zeta^{(2)}+4 v^{2} \zeta^{(2)}=6 v^{2} \zeta^{(1)^{2}},
$$

5 
with the understanding that $v$ is shifted by the amount given by Eq. (2.13). This amount is often small and can be neglected. For example, if $c=2$, and $\Delta p / p=.01, \Delta v=.005$ which is not appreciable unless $2 v$ is very close to integer,

Eq. (2.14) for $\zeta^{(2)}$ can be solved by writing

$$
\begin{aligned}
\zeta^{(2)} & =\sum_{m} \zeta_{m}^{(2)} e^{i A} \\
\zeta_{m}^{(2)} & =\frac{6 v^{2}\left(\zeta^{(1) 2}\right) m}{4 v^{2}-m^{2}} \\
& =\frac{6 v^{2}}{4 v^{2}-m^{2} n_{1}+n_{2}=m} \zeta_{n_{1}}^{(1)} \zeta_{n_{2}}^{(2)}
\end{aligned}
$$

An approximate more usable result can be found in the case where $2 \mathrm{~V}$ is close to an integer $n$. In this case $\zeta_{n}^{(1)}$ given by Eq. (2.11) dominates, and

$$
\begin{aligned}
\zeta_{n}^{(2)} & =\frac{6 v^{2}}{4 v^{2}-n^{2}} 2 \zeta_{n}^{(1)} \zeta_{0}^{(1)}, \\
\zeta_{0}^{(1)} & =\frac{1}{4 v^{2}}\left(-v^{2} b^{4} \Delta R\right)_{0}, \\
& =-\frac{1}{2 v} \mathrm{c} \Delta p / p .
\end{aligned}
$$

$\mathrm{C}$ is the chromaticity $\mathrm{c}=\mathrm{p} \mathrm{d} \nu / \mathrm{dp}$ and $\mathrm{c}=\int \mathrm{ds} \beta \Delta K / 4 \pi$. Terms $1 \mathrm{ike} \zeta_{-n}^{(1)} \zeta_{2 n}^{(1)}$ have been dropped. Thus one finds for $\zeta_{n}^{(2)}$, 


$$
\begin{gathered}
\zeta_{n}^{(2)}=\frac{12 v^{2}}{4 v^{2}-r^{2}} \frac{2 v \Delta v_{2}}{n^{2}-4 v^{2}}\left(-\frac{1}{2 v} c \Delta p / p\right), \\
\tau_{n}^{(2)}=\frac{12 v^{2}}{\left(4 v^{2}-n^{2}\right)^{2}} c \frac{\Delta p}{p} \Delta v_{2}, \\
\zeta_{n}^{(2)}=\frac{3}{4} \frac{1}{(2 v-n)^{2}} \subset \frac{\Delta p}{p} \Delta v_{2} .
\end{gathered}
$$

Note that $\tau_{n}^{(1)}$ is given by

$$
\zeta_{n}^{(1)}=\frac{1}{2} \frac{1}{(n-2 v)} \Delta v_{2}
$$

It may be noted that $\tau_{n}^{(2)}$ is quadratic in $\Delta p / p$ as $\Delta v_{2}$ is linear in $\Delta p / p$. Eq. (2.17) shows that the second order term varies inversely as the square of $(2 v-n)$, the distance from the resonance line.

The variation of $B$ with momentum can be found from the exact relationship

$$
\begin{aligned}
& \frac{\Delta B}{R}=2 \frac{\Delta b}{b}+\left(\frac{\Delta b}{b}\right)^{2}, \\
& \frac{\Delta B}{B}=25+c^{2} .
\end{aligned}
$$

$\zeta$ is given by

$$
\zeta=2\left|\zeta_{n}^{(1)}+\zeta_{n}^{(2)}\right| \cos \left(n A+\delta_{2}\right)
$$

since $\tau_{n}^{(1)}$ and $\zeta_{n}^{(2)}$ have the same phase, the phase of $\Delta v_{2}$ which is denoted by $\delta_{2}$. Thus $\triangle B / B$ is given by 


$$
\begin{gathered}
\frac{\Delta B}{B}=4\left|\zeta_{n}^{(1)}+\zeta_{n}^{(2)}\right| \cos \left(n A+\delta_{2}\right)+4\left|\zeta_{n}(1)\right|^{2} \cos ^{2}\left(n^{A}+\delta_{2}\right) . \\
\zeta_{n}^{(1)}=\frac{1}{2} \frac{1}{(n-2 v)} \Delta v_{2}, \\
\zeta_{n}^{(2)}=\frac{3}{4} \frac{1}{(n-2 v)^{2}} c \frac{\Delta p}{P} \Delta v_{2} .
\end{gathered}
$$

The maximum variation of $B$ with $\Delta p / p$ can then be written as

$$
\begin{aligned}
\left(\frac{\Delta B}{B}\right)_{\max } & =\left(\frac{\Delta B}{B}\right)_{\max }^{(1)}+\left(\frac{\Delta B}{B}\right)_{\max }^{(2)} \\
\left(\frac{\Delta B}{B}\right)_{\max }^{(1)} & =4\left|\zeta_{n}^{(1)}\right|=\frac{2}{|n-2 v|}\left|\Delta v_{2}\right| \\
\left(\frac{\Delta B}{R}\right)_{\max }^{(2)} & =4\left|\zeta_{n}^{(2)}\right|+4\left|\zeta_{n}^{(1)^{2}}\right| \\
& =\frac{3}{(n-2 \nu)^{2}} \mathrm{C} \frac{\Delta p}{P}\left|\Delta v_{2}\right|+\frac{1}{(n-2 v)^{2}}\left|\Delta v_{2}\right|^{2}
\end{aligned}
$$

\section{Comparison of Theory with Computed Results}

During the design study of the CBA, a lattice was considered which has a strong half-integer stop-band at $v=22.5=45 / 2$. The operating $v$-value is $v=$ 22.65. This lattice exhibited a strong dependence of the $B$-function on $\Delta_{p} / p$. $B_{x}$ varied from $R_{x}=67$ at $\Delta_{p} / p=0$ to $R_{x}=150$ at $\Delta_{p} / p=.01$. The dependence of $B_{x}$ on $\Delta p / p$ and on azimuth was computed using the SYNCH program ${ }^{2}$, and the results compared with the theoretical results of the previous section.

For this lattice, the stop band half-wiath at $v=22.5$ was computed to be $\Delta v_{2}=.13$. In order to compute $\Delta B / B$ from $E_{4} \cdot(2.20)$, one needs the chromaticity 
C. A difficulty arises here, because accelerators tend to operate with low value of $c$. The lattice has a $c_{x}=-40$ before the sextupoles are excited which is then reduced to $C_{x}{ }^{2}$ using the sextupoles.

In the SYNCH run, the sextupoles were adjusted to give $C_{x}=1.2$. If one

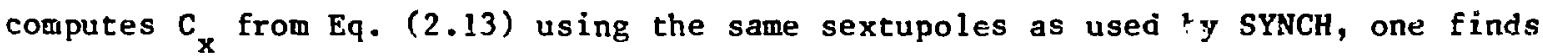
$C_{x}=-5.7$. These two results for $C_{x}$ give considerably different results for: $\zeta_{n}^{(2)}$ in Eq. (2.20) which is proportional to $\mathrm{C}_{\mathrm{x}}$. In what follows, the value $C_{x}=1.2$ found by the SYNCH program was used. One then finds from Eq. (2.20)

$$
\begin{aligned}
& \zeta_{n}^{(1)}=-.22 \\
& \zeta_{n}^{(2)}=.013
\end{aligned}
$$

The $\zeta_{n}^{(1)}$ term gives a linear variation in $\beta$ of $4 \zeta_{n}^{(1)}$ or $A B=59$ for $\Delta_{p} / p=$ .01 , and a quadratic variation in $\Delta B / B$ of $4 /\left.\zeta_{n}^{(1)}\right|^{2}$, or $\Delta B=13$. The $\zeta_{n}^{(2)}$ term gives a quadratic variation in $\Delta B / 6$ of $4 \zeta_{n}^{(2)}$ or $\Delta B=3.5$.

The 1 inear result predicts that $\triangle B / B$ will vary azimuthally like cos $2 \psi$ where $\psi$ is the betatron phase. In the lattice considered here, $\Delta U$ fc: one cell is $\pi / 2$ and the variation of $\triangle B / B$ with $A$ has a period of 2 cells. The non-1inear term in Eq. $(2.2), 4\left|\zeta_{n}^{(1)}\right|^{2} \cos ^{2}\left(n^{A}+\delta_{2}\right)$ changes this $A$-dependence of $\Delta B / B$ adding a term that goes like $\cos ^{2} 2 \psi$. The following table shows the $A$ variation of $B_{x}$ as given by the theory and by the SYNCH program.

\begin{tabular}{cccc}
$U$ & 0 & $\pi / 2$ & $\pi$ \\
\hline$B_{x}{ }^{-T h e o r y}$ & 143 & 18 & 143 \\
$B_{x}{ }^{-S Y N C H}$ & 147 & 30 & 147
\end{tabular}

The agreement between theory and SYNCH at $W=\pi / 2$ is only fair, but $\beta_{x}$ is stiall there and large errors may be expected. There appears to be some evidence for the existance of the $\cos ^{2} 2 \psi$ term. 
The linear result predicts that $\Delta \beta / \beta$ will vary linearly with $\Delta p / p$. The SYNCH results indicate an appreciable $(\Delta p / p)^{2}$ term. This is illustrated by the folluwing table.

\begin{tabular}{cccc}
$\Delta p / p$ & .01 & 0 & -.01 \\
\hline$B_{x}-$ Theory & 143 & 67 & 25 \\
$B_{x}$-SYNCH & 147 & 67 & 30
\end{tabular}

4. Correction of $B(p)$ in Proton Storage Rings

According to linear theory the variation of $\beta$ with momentum given by (see Eq. (2.a)).

$$
\frac{\Delta B}{B}=-\frac{1}{2 \sin 2 \pi \nu} \int d s_{0} \cos \left(2 \pi v-\left|\psi(s)-\psi\left(s_{0}\right)\right|\right) B\left(s_{0}\right) A K\left(s_{0}\right)
$$

For $\Delta B \mathbf{x}^{/ B} \mathbf{x}^{\prime}$

$$
\Delta K_{x}=\left(-G \Delta_{p} / p+2 B_{2} x_{p} \Delta_{p} / p\right) / B \rho,
$$

and for $\Delta S_{x} / B, B+B$ and $\Delta k_{y}=-\Delta k_{x}$.

The sextupoles must be chosen not on $1 y$ to minimize the $B$-variation at each magnet location but also to give the proper chromaticity, $c_{x}, c_{y}$ which is given by

$$
\mathrm{C}=\frac{1}{4 \pi} \int \mathrm{d} s \Delta \mathrm{K}
$$

$\Delta \mathrm{K}_{\mathrm{x}}$ and $\Delta \mathrm{K}_{\mathrm{y}}$ are given above.

One needs two families of sextupoles to determine $c_{x}$ and $c_{y}$. The problem of using linear theory to minimize $\Delta \beta / B$ and get the correct chromaticity has been treated by many people including those listed in Reference (3). If one is fortunate in the choice of the location of the sextupoles, it may be possible to 
minimize $\Delta B_{x} / B_{x}$ and $\Delta \beta_{y} / B_{y}$ and correct $C_{x}$ and $C_{y}$ with 4 families of sextupoles. It is more likely that 6 or more families of sextupoles would be required.

For the lattice described in Section 3, the choice of the location of the sextupole families was determined by the location of the sextupole coils already in the accelerator for the purpose of correcting the chromaticity. These sextupole coils were arranged in four families to $\operatorname{correct} \beta_{x}(p)$, and $\operatorname{not}_{\mathbf{y}}(p)$, and to correct both horizontal and vertical chromaticities, $\mathrm{C}_{\mathrm{x}}$ and $\mathrm{C}_{\mathrm{y}}$. Two groups of the horizcntal sextupole coils were observed to be located at the peaks and valleys of the azimuthal variation of $B_{x}$ at $\Delta_{p} / p=.01$, and these two families were used to correct $B_{x}(p)$. The remaining horizontal sextupole coils was a third family, and the vertical sextupole coils was a fourth family.

Having chosen the location of the sextupoles and the number of sextupole families, one could find the strength of the sextupoles by computing $\Delta B / B$ at $\mathrm{var}-$ ious suitable observation points using the linear result Eq. (4.1), and then minimizing

$$
I=\frac{1}{k_{\max }} \sum_{k}\left(\frac{\Delta B}{B}\right)_{k}^{2},
$$

subject to the chromaticity conditions

$$
\begin{aligned}
& c_{x}=c_{x, 0}, \\
& c_{y}=c_{y, 0},
\end{aligned}
$$

where $C_{x}, C_{y}$ are computed using Eq. $(4.2)$, and $c_{x, 0}, c_{y, 0}$ are the desired chromaticities. The index $k$ enumerates the places where $\triangle B / B$ is observed; $k_{\max }$ is the total number of observation points.

The obvious defect in the above procedure is that $\triangle B / B$ may have an appreciable non-linear part, as given by Eq. $(2.20)$, which is not included in 
the above formulation. Nevertheless, minimizing just the linear part of $\Delta B / B$ may in some cases give reasonable results. If the $\Delta \beta / B$ variation is dominated by a nearby stopband of half-width $\Delta v_{2}$, then minimizing the linear part of $\Delta B / \beta$ may result in a selection of the sextupole strengths that also reduces $\Delta \nu_{2}$, thus reducing the non-linear part of $\Delta \beta / \beta$. However computer studies carried out for the lattice discussed in Section 3 shows that Eq. (4.3a) sometimes leads to solutions that do not reduce $\Delta \nu_{2}$ and which actually increase the non-linear part of $\triangle B / B$.

A procedure we have used to avoid the above difficulty is to minimize

$$
\left.I=\frac{1}{k_{\max }} \sum_{k}\left(\frac{\Delta \beta^{(1)}}{\beta}\right)^{2}+\lambda^{2}\left|\Delta v_{2}\right|^{2}\right)
$$

where $\Delta \beta^{(1)} / \beta$ is the linear part of $\Delta \beta / \beta$ given by $E q .(4.1) ; \Delta \nu_{2}$ is the iialfwidth of the nearby half-integer stopband and is given by

$$
\Delta v_{2}=\frac{1}{4 \pi} \int_{d s} B_{x} \Delta k_{x} e^{-i n \theta} x
$$

$\lambda$ has to be chosen so as to make the $\lambda^{2}\left|\Delta v_{2}\right|^{2}$ term in Eq. (4.4a) approximately equal to the non-linear contribution to $\Delta B / B$; that is $\Delta \beta^{(2)} / \beta \simeq \lambda\left|\Delta \nu_{2}\right|$. Using Eq. (2.20), a rough result for $\lambda$ is

$$
\lambda=\left|\frac{3}{\left(n-2 v_{x}\right)^{2}} c_{x} \Delta_{p} / p\right|+\frac{1}{2\left(n-2 \nu_{x}\right)^{2}}\left|\Delta v_{2}^{(0)}\right|
$$

$\Delta v_{2}{ }^{(0)}$ is the stopband width before exciting the sextupoles.

In the above formulation, we are only correcting $\Delta \beta_{x} / \beta_{x}$ and not $\Delta \beta_{y} / \beta_{y}$ and have assumed only one stop-band dominates. If more than one stopband plays a role, or one wants to correct $\Delta \beta_{y} / R_{y}$ as well, then one would need to add the ap- 
propriate terms to Eq. (4.4) giving the contributions of $\Delta B_{y} / B_{y}$ and of the other relevant stop-bands. The choice of $\lambda$ can be adjusted empirically by computing $\Delta \beta^{(2)} / \beta$ using Eq. (2.21) and comparing it with $\Delta \beta^{(1)} / \beta$.

The formulation given by Eq. (4.4) was tested by computing the sextupole strengths required to correct the $B(p)$ variation in the lattice described in Section 3. This lattice has a large $\beta$-variation as $\beta_{x}$ changes from $\beta_{x}=67$ at $\Delta p / p=0$ to $B_{x}=147$ as $\Delta_{p} / p=.01$, a relative variation of $\Delta B / B \simeq 100 \%$. Four families of sextupoles were used and results were computed using the SYNCH program, and using Eq. (4.4). A comparison of the sextuple strengths found to correct the $B_{x}(p)$ variation is given in the following tabli.

\begin{tabular}{rrr} 
& Theory & SYNCH \\
\hline $\mathrm{b}_{2}(1)$ & 3.949 & 3.319 \\
$\mathrm{~b}_{2}(z)$ & 1.902 & 1.869 \\
$\mathrm{~b}_{2}(3)$ & 2.912 & 2.594 \\
$\mathrm{~b}_{2}(4)$ & -2.048 & -1.911 \\
\hline
\end{tabular}

$\mathrm{b}_{2}$ is the sextupole strength, $\mathrm{b}_{2}=\mathrm{B}^{\prime \prime} / 2 \mathrm{~B}$, and is given in units of $10^{-4} \mathrm{~cm}^{-?}$. Using the SYNCH program, the $B_{x}$ variation was reduced from $67 \rightarrow 147$ before correction to $67 \rightarrow 75$ after correction. Using Eq. 4.4 , the $B_{x}$ variation after correction was found to be $67+74$. 


\section{References}

1. E.D. Courant and H. Snyder, Annals of Physics, $\underline{3}$, No. 1, (1958).

2. A.A. Garren and A.S. Kenney (unpublished), (1968).

3. H. Wiedeman, PEP-220, (1976).

B. Autin and A. Verdier, CERN ISR-LTD/76-14, (1976).

G. Guignard, CERN ISR-TH/82-14 (1982).

B.W. Montague, LEP Note 165, (1979). 